\title{
Neutrophilic bacterial meningitis: pathology and etiologic diagnosis of fatal cases
}

\author{
Jeannette Guarner ${ }^{1}$, Lindy Liu², Julu Bhatnagar², Tara Jones², Mitesh Patel², \\ Marlene DeLeon-Carnes ${ }^{2}$ and Sherif R Zaki ${ }^{2}$ \\ ${ }^{1}$ Department of Pathology and Laboratory Medicine, Emory University Hospital, Atlanta, GA, USA and \\ ${ }^{2}$ Infectious Diseases Pathology Branch, Centers for Disease Control and Prevention, Atlanta, GA, USA
}

\begin{abstract}
The frequency of fatalities due to acute bacterial meningitis has decreased significantly due to vaccinations, early diagnoses, and treatments. We studied brain tissues of patients with fatal neutrophilic meningitis referred to the Centers for Disease Control for etiologic diagnosis from 2000-2009 to highlight aspects of the disease that may be preventable or treatable. Demographic, clinical, and laboratory data were extracted from records. Of 117 cases in the database with a diagnosis of meningitis or meningoencephalitis, 39 had neutrophilic inflammation in the meninges. Inflammatory cells infiltrated the superficial cortex in 16 of $39(41 \%)$ cases. Bacteria were found using Gram and bacterial silver stains in $72 \%$ of cases, immunohistochemistry in $69 \%$ (including two cases where the meningococcus was found outside the meninges), and PCR in $74 \%$. Streptococcus pneumoniae was the cause of the meningitis in 14 patients and Neisseria meningitidis in 9. In addition, Streptococcus spp. were found to be the cause in six cases, while Staphylococcus aureus, Staphylococcus spp., Enterococcus spp., and Fusobacterium were the cause of one case each. There were six cases in which no specific etiological agent could be determined. The mean age of the patients with S. pneumoniae was 39 years (range 0-65), with $N$. meningitidis was 19 years (range 7-51), whereas that for all others was 31 years (range $0-68$ ). In summary, our study shows that $S$. pneumoniae continues to be the most frequent cause of fatal neutrophilic bacterial meningitis followed by $\boldsymbol{N}$. meningitidis, both vaccine preventable diseases.

Modern Pathology (2013) 26, 1076-1085; doi:10.1038/modpathol.2013.30; published online 5 April 2013
\end{abstract}

Keywords: bacterial meningitis; diagnosis; etiology

Acute bacterial meningitis has been defined clinically by the classic triad of fever, neck stiffness, and altered mental status; however, the sensitivity of this combination of signs and symptoms is $\sim 40 \%$ and the specificity of each of these alone ranges from 15$60 \%{ }^{1,2}$ Thus, laboratory parameters have been added to aid in the diagnosis of acute meningitis, and require a cerebrospinal fluid (CSF) showing elevated white blood cells counts (between 100 and 10000 cells $/ \mathrm{mm}^{3}$ ) with $80-95 \%$ of cells being neutrophils, elevated protein, and decreased glucose. Although, the overall sensitivity of Gram stains on the CSF ranges from 10 to $90 \%$ depending on the organism causing the infection and the amount of organisms present in the fluid, determining the Gram staining characteristics of the organism

Correspondence: Dr J Guarner, MD, Department of Pathology and Laboratory Medicine, Emory University Hospital, 1364 Clifton Road NE, Room F149B, Atlanta, GA 30322, USA.

E-mail: jguarne@emory.edu

Received 13 September 2012; revised 15 December 2012; accepted 17 December 2012; published online 5 April 2013 in the CSF is of great use for starting empiric treatment. ${ }^{3}$ Finding neutrophils and bacteria in the CSF should reflect what is observed in the meninges of patients that die of acute bacterial meningitis.

In general, the frequency of fatalities due to acute bacterial meningitis has decreased significantly in the past two decades since the introduction of the Haemophilus influenzae type b vaccine. However, mortality reported for patients with bacterial meningitis ranges between 9 and $35 \%$ depending on the bacterium causing the meningitis, the prevalence of the different pathogens in particular populations, having a diagnosis, and the healthcare that can be provided to the patients. ${ }^{4-9}$ Worldwide, Streptococcus pneumoniae, Neisseria meningitidis, Streptococcus agalactiae, Escherichia coli, and Listeria monocytogenes are the most frequent etiologic agents of community-acquired bacterial meningitis. ${ }^{4-9}$ Staphylococcus aureus is the most frequent pathogen in patients that have undergone intracranial surgery. ${ }^{6}$ Surveillance data based on cultures from eight areas participating in the US Emerging Infections Programs Network in 
the 1998-2007 period showed that S. pneumoniae caused meninigitis in $58 \%$ of cases and accounted for $71 \%$ of the fatalities. ${ }^{10}$ Other bacteria causing meningitis in this surveillance study included group B streptococci (18\%), N. meningitidis (14\%), $H$. influenzae $(7 \%)$, and L. monocytogenes (3\%). Yet, a specific bacterium is not detected in all patients with clinical and laboratory evidence of meningitis since CSF cultures have been shown to be positive in $13-96 \%$ of cases depending on timing of obtaining the CSF and initiation of antibiotics. ${ }^{3}$

Post-mortem studies that compare the pathology of the different bacteria causing acute meningitis in the antibiotic era are important to better define the different pathogenic mechanisms and potential preventive, and treatment aspects of this devastating disease. Previous pathologic studies of fatal cases with acute meningitis caused by $S$. pneumoniae have shown neutrophilic inflammation of the subarachnoid space with edema, inflammation of vessels, and necrosis in the adjacent brain parenchyma. ${ }^{11,12}$ The inflammation of parenchymal and subarachnoid vessels has been described as arteritis and phlebitis with thromboses. In contrast, normal white blood cell counts in the CSF can occur in up to $10 \%$ of patients and have been associated with a worse prognosis. ${ }^{1}$

The Infectious Diseases Pathology Branch at the United States Centers for Disease Control and Prevention receives tissues from cases that are referred, because no pathogen was found but an infectious agent is suspected or for the confirmation of a pathogen of public health significance. The objective of this study was to identify pathogenic organisms and their frequency in fatal meningitis cases with predominant neutrophilic inflammation that were referred over a 10 year period to Infectious Disease Pathology Branch. In addition, we studied the pathologic features of these cases to better define the pathogenesis of fatal neutrophilic bacterial meningitis.

\section{Materials and methods}

The Infectious Disease Pathology Branch database was used to identify cases with a diagnosis of meningitis or meningoencephalitis during the first 10 years of the 21st century (2000-2009). These cases were submitted for consultation to Infectious Disease Pathology Branch from local state health departments and medical examiners. Demographic and pertinent clinical and laboratory data, including CSF and blood cultures submitted varied in completeness. One hundred and seventeen cases were identified using the database. In four cases the material was not available for study since slides had been returned to the primary sender, thus hematoxylin and eosin (H\&E) slides of 113 cases were reviewed. In three cases no meninges were available for study. Of the remaining 110 cases, 48 had perivascular cuffing, glial nodules, and mononuclear mild meningeal inflammation, 19 had granulomatous inflammation, and 4 had meningeal hemorrhage. These 71 cases were considered to have either viral meningoencephalitis, tuberculosis or fungal central nervous system infections, and hemorrhages, thus they were excluded from the study. The total number of cases with predominantly neutrophilic meningitis that were included in the study was 39 .

To define whether there was a difference between the ages of the patients that had pneumococcal infection compared with those having meningococcal infection, a two-tailed $t$-test was applied using the online $t$-test calculator: http://www.graphpad. com/quickcalcs/ttest2/ and values $<0.05$ were considered statistically significant.

Pathologic features assessed with H\&E stains included the amount of inflammation in the meninges, which was graded as abundant if the inflammation expanded the meninges, moderate if the inflammation did not expand the meninges, or mild if scattered neutrophils were observed in the meninges, and the presence of inflammation in the adjacent cortex, thrombosis, hemorrhage, and necrosis. In general, cases were first stained with Gram (Brown-Brenn or Lillie Twort) and bacterial silver impregnation (Steiner or Warthin Starry) stains, and depending on the result appropriate immunohistochemistry and PCR assays were performed. Thus, cases with gram-positive cocci were tested using immunohistochemistry for Streptococcus spp, while those with gram-negative cocci were tested using the immunohistochemistry for $N$. meningitidis. Cases with gram-variable bacteria were tested using immunohistochemistry for Streptococcus spp. and $N$. meningitidis. PCR was used for confirmation of immunohistochemistry results or to further investigate the possible organism causing the neutrophilic meningitis.

\section{Immunohistochemistry Assays}

Immunohistochemical assays for infectious agents (Streptococcus species, S. aureus, Streptococcus pyogenes, and $N$. meningitidis) have been previously described, ${ }^{13-18}$ although modifications of portions of the procedures have occurred through the years encompassed in the study. In summary, assays were performed on deparaffinized, rehydrated $3-\mu \mathrm{m}$ tissue sections. Proteinase $\mathrm{K}$ (Roche, Indianapolis, IN, USA) digestion was carried out for slides treated with all antibodies. Incubation with a primary antibody and colorimetric detection with either the Universal LSAB 2 System-Alkaline Phosphatase kit (Dako, Carpinteria, CA, USA) or the Ultra Vision LP Detection System/AP Polymer \& Fast Red Chromogen (Lab Vision, Freemont, CA, USA) were performed in the Dako autostainer or the Lab Vision Autostainer 360. Primary antibodies included: (1) a 
polyclonal rabbit antibody against Streptococcus spp. (Meningitis and Vaccine Preventable Diseases Branch, CDC), (2) a polyclonal horse antibody against $N$. meningitidis (Center for Biologics Evaluation and Research, Food and Drug Administration), (3) a polyclonal rabbit antibody against $S$. aureus (Meridian Life Science, Saco, ME, USA), and (4) a monoclonal $S$. aureus antibody (QED Bioscience, San Diego, CA, USA). Table 1 shows the dilutions each antibody was used and cross-reactivity testing that has been performed. Sections were counterstained with Mayer's hematoxylin (Fisher Scientific, Pittsburgh, PA, USA). Positive controls for the primary antibodies included formalin-fixed, paraffin-embedded bacterial cultures, and cases with known bacterial infections. Negative controls consisted of sequential casepatient tissue sections incubated with normal rabbit, mouse, or horse serum as the primary antibody.

\section{DNA Extraction and PCR Assays}

DNA was extracted from $10-\mu \mathrm{m}$ formalin-fixed, paraffin-embedded tissue sections using the QIAamp DNA Mini Kit (Qiagen, Valencia, CA, USA), following the tissue extraction protocol as described previously. ${ }^{19}$ Microcentrifuge tubes lacking tissue sections (extraction blanks) were included in each extraction run to ensure no reagent or crosscontamination occurred during the extraction procedure. To evaluate the quality of DNA extract, each sample was tested for the amplification of the house-keeping genes human glyceraldehyde-3-phosphate dehydrogenase (GAPDH) and $\beta$-globin. ${ }^{20,21}$

The DNA extracts were evaluated by conventional PCR assays for $S$. pneumoniae, ${ }^{13,20}$ N. meningitidis, ${ }^{14,22}$ Streptococcus species, and by paneubacterial $\mathrm{PCR}^{23}$ using the High-Fidelity

Table 1 Antibodies used in immunohistochemical assays

\begin{tabular}{|c|c|c|c|}
\hline Antibody against & Animal source & Dilution & Reference \\
\hline S. pneumoniae ${ }^{\mathrm{a}}$ & Rabbit & $1: 3000$ & 13 \\
\hline N. meningitidis ${ }^{\mathrm{b}}$ & Horse & $1: 500$ & 14 \\
\hline S. aureus & Rabbit & $1: 500$ & 15 \\
\hline S. aureus ${ }^{\mathrm{d}}$ & Mouse & $1: 100$ & \\
\hline
\end{tabular}

a The $S$. pneumoniae antibody does not react with group A streptococcus (GAS), group B streptococcus (GBS), S. aureus, $B$. anthracis, F. tularensis, L. pneumophila, Leptospira spp., $H$. influenzae, $K$. pneumoniae, $M$. pneumoniae, $M$. tuberculosis, $N$. meningitidis, or $Y$. pestis. This antibody reacts with several streptococci including $S$. mitis and $S$ suis.

${ }^{b}$ The $N$. meningitidis antibody does not react with GAS, S. aureus, L. monocytogenes, $B$. anthracis, $F$. tularensis, $H$. influenzae, $M$. pneumoniae, Pseudomonas spp., or $R$. rickettsii.

${ }^{\mathrm{C}}$ The $S$. aureus antibody does not react with GAS, GBS, $S$. pneumoniae, B. anthracis, Clostridium spp., K. pneumoniae, Pseudomonas spp., or N. meningitidis.

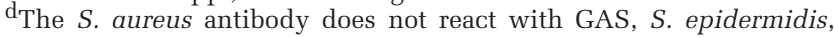
Streptococcus viridans group, $H$. influenzae, $S$. pneumoniae, Bordetella spp., Yersinia pestis, Klebsiella spp., E. coli, B. henselae, E. faecalis, or $P$. aeruginosa.
PCR kit (Roche Diagnostics, Indianapolis, IN). Primer sequences, target genes, PCR annealing temperatures, and amplification product sizes of conventional PCR assays are summarized in Table 2. In addition, DNA extracts were also tested by staphylococcal real-time multiplex PCR assay targeting the Panton-Valentine leukocidin gene and toxic shock syndrome toxin 1 gene. PCR assays were performed using the previously published primers and probes $^{22,23}$ and the QuantiTect Multiplex PCR Kit (Qiagen) on the Stratagene Mx3000P QPCR system.

Each PCR run included, a positive control (DNA extracted from formalin-fixed, paraffin-embedded bacterial cultures), an extraction negative control (extraction blank), and a water control (nontemplate DNA) between two adjacent patient samples. Additionally, universal contamination precautions, including the physical separation of the postamplification procedures from the extraction and PCR reagent preparation areas, use of filtered pipette tips, and UV irradiation of PCR trays and tubes, were carried out.

\section{Sequencing of Amplification Products}

The amplification products of conventional PCR assays were visualized by using $2 \%$ agarose gels containing ethidium bromide. For positive samples, the remaining reaction $(40 \mu \mathrm{l})$ was loaded into $1.8 \%$ agarose gels and bands of the appropriate size were cut out. DNA was recovered from the excised fragments by using a QIAquick Gel Extraction Kit (Qiagen, Valencia, CA, USA). Automated nucleotide sequencing was performed for all the positive samples by using a CEQ DTCS Quick Start Kit on the CEQ 8000 XL Genetic Analysis System sequencer (Beckman Colter, Fullerton, CA, USA). A search for homologies to known sequences was done using the Basic Local Alignment Search Tool (BLAST) at http://www.ncbi.nlm.nih.gov/BLAST.

\section{Results}

There were 16 pediatric patients ranging from 1 week to 18 years (mean 10) and 21 adults ranging from 19 to 68 years (mean 44). For two patients we did not receive information regarding age. There were 20 $(53 \%)$ females. Four patients were from Taiwan and the remaining from the US. S. pneumoniae was the cause of the meningitis in $14(36 \%)$ patients; in 13 of these patients $S$. pneumoniae was detected using immunohistochemistry and PCR performed on formalin-fixed, paraffin-embedded tissues. In one patient, the diagnosis was based on PCR performed on a post-mortem CSF sample in the facility where the patient was seen originally. $N$. meningitidis was the cause of the meningitis in nine $(23 \%)$ patients (including two patients from Taiwan); in seven of these patients $N$. meningitidis was detected in the 
Table 2 Primers used in conventional PCR assays

\begin{tabular}{|c|c|c|c|c|}
\hline PCR/primers & Sequence $\left(5^{\prime}-3^{\prime}\right)$ & Gene target & Product size & Ann. temp. \\
\hline \multicolumn{5}{|c|}{ N. meningitidis $P C R$} \\
\hline Omp56 & CCAGCGGTATTGTTTGGTGGT & $\operatorname{ctr} A$ & $177 \mathrm{bp}$ & $55^{\circ} \mathrm{C}$ \\
\hline Omp212 & CAGGCGGCCTTTAATAATTTC & & & \\
\hline \multicolumn{5}{|c|}{ S. pneumoniae nested-PCR } \\
\hline Ia & ATTTCTGTAACAGCTACCAACGA & ply & $348 \mathrm{bp}$ & $55^{\circ} \mathrm{C}$ \\
\hline $\mathrm{Ib}$ & GAATTCCCTGTCTTTTCAAAGTC & & & \\
\hline IIa & CCCACTCTTCTTGCGGTTGA & ply & $208 \mathrm{bp}$ & $55^{\circ} \mathrm{C}$ \\
\hline IIb & TGAGCCGTTATTTTTTCATACTG & & & \\
\hline \multicolumn{5}{|c|}{ Streptococcus spp. PCR } \\
\hline S16-F & CAGATGGACCTGCGTTGTATTAGC & 16S rRNA & $400 \mathrm{bp}$ & $57^{\circ} \mathrm{C}$ \\
\hline S16-R & GGTTAAGCCACAGCCTTTAACTTC & & & \\
\hline \multicolumn{5}{|c|}{ Paneubacteria PCR } \\
\hline FD1 & AGAGTTTGATCCTGGCTCAG & 16S rRNA & $529 \mathrm{bp}$ & $59^{\circ} \mathrm{C}$ \\
\hline $532 \mathrm{R}$ & TACCGCGGCTGCTGGCAC & & & \\
\hline \multicolumn{5}{|c|}{ Internal controls (GAPDH and $\beta$-globin) PCR } \\
\hline$\beta$-globin-F & GCCAGTGCCAGA AGAGCCAA & $\beta$-globin & $500 \mathrm{bp}$ & $60{ }^{\circ} \mathrm{C}$ \\
\hline$\beta$-globin-R & TTAGGGTTGCCCATAACAGC & & & \\
\hline GAPDH-F & AGGTGGAGCGAGGCTAGC & GAPDH & $200 \mathrm{bp}$ & \\
\hline GAPDH-R & TTTTGCGGTGGA AATGTCCT & & & \\
\hline
\end{tabular}

Abbreviations: ann. temp., annealing temperature; ctrA, outer membrane protein; GAPDH, glyceraldehyde-3-phosphate dehydrogenase; ply, pneumolysin.

meninges using immunohistochemistry and PCR on formalin-fixed, paraffin-embedded tissues, while in two the meningococi were observed in other tissues including the liver, spleen, and others. The age of the patients with $N$. meningitidis was significantly lower (mean 19 years, range 7-51) compared with that of the patients with $S$. pneumoniae meningitis (mean 39 years, range $0-65)(P=0.025)$. In six $(15 \%)$ patients, Streptococcus spp. was determined to be the cause of the meningitis (two by PCR, two by immunohistochemistry, and two by immunohistochemistry and PCR). There were also one case of each $S$. aureus (by immunohistochemistry and PCR), Staphylococcus spp. (by PCR), Enterococcus spp. (on a post-mortem culture of blood and CSF performed in the facility where the patient was seen originally), and Fusobacterium necrophorum (identified by paneubacterial PCR and sequencing). Lastly, there were six $(15 \%)$ cases, in which no specific etiologic agent could be determined, three cases had grampositive cocci, two cases had gram-negative coccobacilli, and the last case had gram-variable cocci.

In $68 \%$ of cases, one or two blocks of the brain tissue were sent for study with an average of three blocks per case. All the cases had meninges and underlying parenchyma except for one, in which only meninges were sent. Overall, severe neutrophilic meningeal inflammation (Figure $1 \mathrm{a}$ and Figure 2a) was present in $25(64 \%)$ cases, $8(20 \%)$ showed moderate inflammation, and 6 (15\%) showed mild inflammation. Hemorrhage into the meninges was found in seven (18\%) cases. Inflammation in the adjacent cortex (Figure 1a) was found in $16(42 \%)$ cases, while hemorrhage into the parenchyma in 7 (18\%) cases, with 4 of these cases having hemorrhage in both the meninges and parenchyma. Thrombosis was observed in one patient with $S$. pneumoniae (Figure 1b) and in two patients where an etiologic agent was not identified. Necrosis was present in one patient with S. pneumoniae and one patient with Streptococcus spp., while leukocytosis in blood vessels was observed in two patients with $N$. meningitidis. Table 3 summarizes special stains, immunohistochemistry, PCR on formalin-fixed, paraffinembedded material, and postmortem culture results. Gram-positive cocci (Figure 1c) were found in 21 $(54 \%)$ cases, gram-negative cocci in $4(10 \%)$, and gram-negative bacilli or coccobacilli in $3(8 \%)$ cases. The Gram stain was concordant with the bacteria found by other methods in $24(61 \%)$ patients, while in $15(39 \%)$ patients the Gram stain was not contributory. Bacterial silver impregnation stains showed bacteria (Figure 1d) concordant to that found by other methods in $14(36 \%)$ cases, but in $25(64 \%)$ cases a variety of cellular elements such as neurosecretory granules and neurofibrils created a background that did not allow for adequate interpretation.

Organism diagnosis to the genus level was achieved using formalin-fixed, paraffin-embedded material in $31(79 \%)$ cases. Immunohistochemistry identified an organism in 27 (69\%) cases. Meningococci were observed in the meningeal inflammatory infiltrate in all cases except in two patients with $N$. meningitidis where the organisms were only found in other tissues (liver, spleen, heart, and kidney). Immunohistochemistry assays demon- 

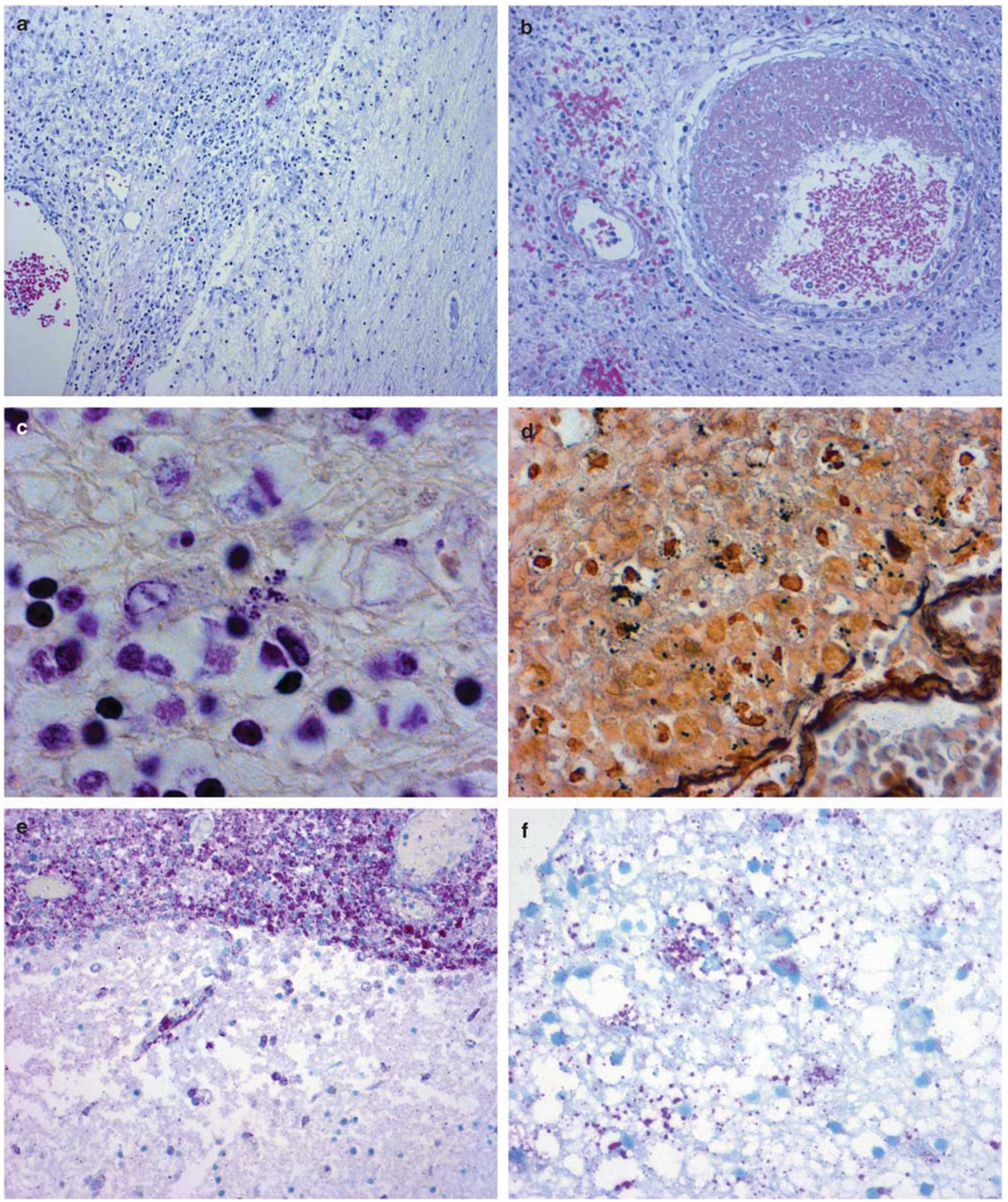

Figure 1 Neutrophilic meningitis caused by S. pneumoniae. (a) Meningeal inflammation spilling into the cortex (hematoxylin and eosin $(\mathrm{H} \& \mathrm{E})$ stain, original magnification $\times 25)$. (b) Thrombosis of vessel in cortex (H\&E stain, original magnification $\times 50$ ). (c) A collection of primarily gram-positive cocci in the meninges (Brown-Brenn Gram stain, original magnification $\times 250$ ). (d) Scattered cocci in the meninges (Steiner silver impregnation stain, original magnification $\times 157.5$ ). Panels $\mathbf{e}$ and $\mathbf{f}$ show abundant cocci and bacterial antigens in the meninges (immunohistochemical assays with the $S$. pneumoniae polyclonal antibody detected with naphthol fast red; original magnification $(\mathbf{e}): \times 50,(\mathbf{f}): \times 100)$. 
strated intact bacteria (cocci) inside cells and in the extracellular spaces as well as bacterial antigens (Figures 1e and f, and Figure 2b). Tissue PCR identified an organism to the genus level in 29 $(74 \%)$ cases and to the species level in $24(61 \%)$. Sequencing the tissue PCR product was able to define the species in 24 cases: $S$. pneumoniae (13 cases), N. meningitidis (all 9 cases), S. aureus (1 case), and $F$. necrophorum (1 case). Tissue PCR followed by sequencing determined the genus but not the species in 5 cases: Staphylococcus sp. (1 case) and Streptococcus species (4 cases, 2 of which had $99 \%$ identity with mitis group streptococci and 1 had 99\% identity with Streptococcus suis and Streptococcus bovis). PCR in tissue was not able to detect an organism in 10 cases (26\%) but in 2 of these cases immunohistochemistry showed streptococci, 1 case had a positive $S$. pneumoniae PCR result from the originating facility, and 1 had grown Enterococcus at autopsy in the originating facility. There were five cases, in which the only diagnosis that could be made was the presence of gram-positive cocci (three cases) or gram-negative coccobacilli (two cases) in the meninges. Lastly, there was a case, in which the diagnosis of acute neutrophilic meningitis was made but organisms were not observed.

\section{Discussion}

This series of cases with fatal neutrophilic bacterial meningitis showed that special stains, immunohistochemistry, and PCR can detect an etiologic agent in over $75 \%$ of cases. The bacteria, for the most part, were observed in the meninges by using Gram and

Table 3 Correlation of etiologic agent and number of cases with a positive tissue diagnostic method

\begin{tabular}{|c|c|c|c|c|c|c|}
\hline \multirow[t]{2}{*}{ Organism } & \multicolumn{4}{|c|}{ Diagnostic method used in the tissues } & \multirow[t]{2}{*}{ Comments } & \multirow{2}{*}{$\begin{array}{l}\text { Total } \\
\text { cases }\end{array}$} \\
\hline & $\begin{array}{l}\text { Gram } \\
\text { stain }^{\mathrm{a}}\end{array}$ & $\begin{array}{l}\text { Silver } \\
\text { stain }^{\mathrm{a}}\end{array}$ & IHC & $\begin{array}{l}\text { PCR on } \\
\text { FFPE }\end{array}$ & & \\
\hline S. pneumoniae & 10 & 5 & 13 & 13 & Post-mortem CSF sample was PCR positive in one case & 14 \\
\hline Streptococcus spp. & 5 & 4 & 4 & 4 & $\begin{array}{l}\text { PCR determined that } 2 \text { were in the mitis group } \\
\text { streptococci; } 1 \text { in Streptococcus suis/bovis; } 1 \text { in } \\
\text { Streptococcus spp. }\end{array}$ & 6 \\
\hline N. meningitidis & 4 & 3 & 9 & 9 & $\begin{array}{l}\text { Gram stain was suspicious in } 2 \text { cases; } 2 \text { cases had IHC } \\
\text { positivity in other tissues }\end{array}$ & 9 \\
\hline Staphylococci & 2 & 1 & 1 & 2 & S. aureus (one case); Staphylococcus spp. (one case) & 2 \\
\hline Enterococcus spp. & 1 & 0 & 0 & 0 & Post-mortem blood and CSF cultures with Enterococcus & 1 \\
\hline Fusobacterium necrophorum & 1 & 1 & 0 & 1 & & 1 \\
\hline Gram-positive cocci & 3 & 3 & 0 & 0 & & 3 \\
\hline Gram-negative cocobacilli & 2 & 2 & 0 & 0 & & 2 \\
\hline Unknown & 0 & 0 & 0 & 0 & & 1 \\
\hline Total & 28 & 19 & 27 & 29 & & 39 \\
\hline
\end{tabular}

Abbreviation: CSF, cerebrospinal fluid.

${ }^{\text {a }}$ Stain concordant with diagnosis.
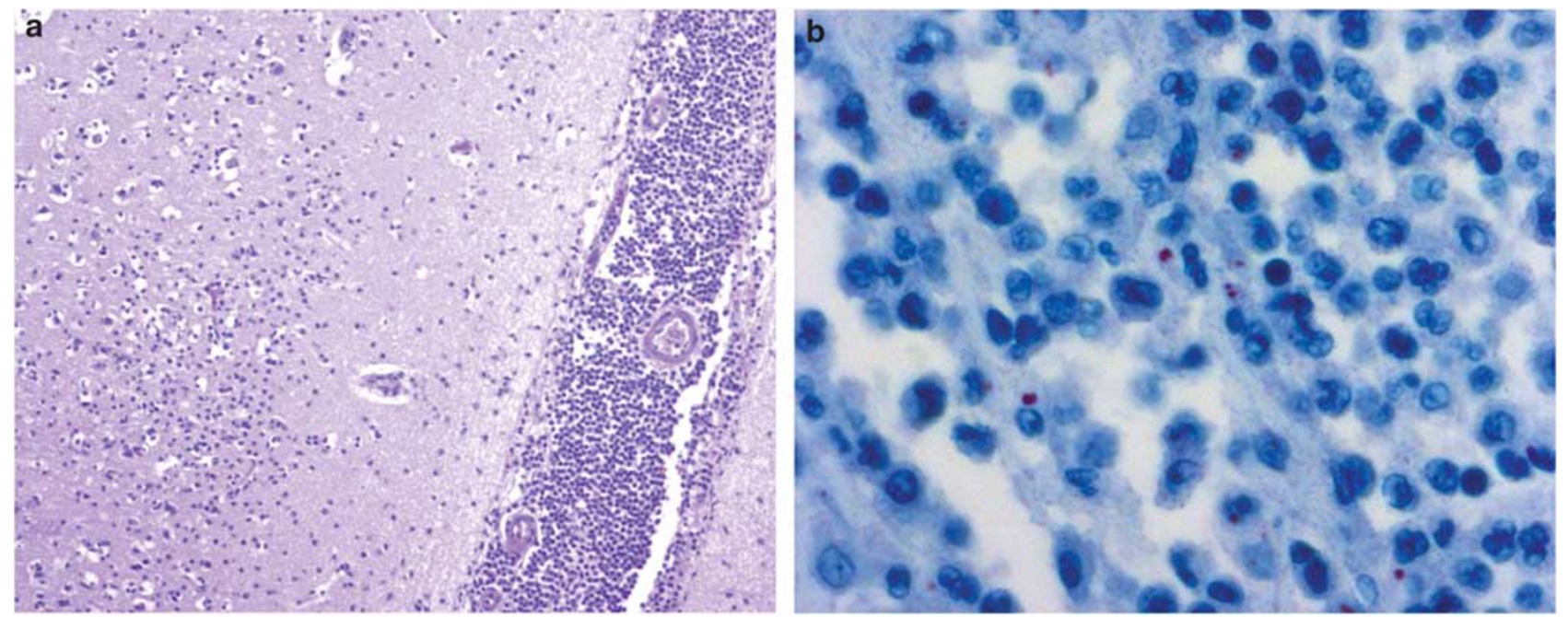

Figure 2 Neutrophilic meningitis caused by N. meningitidis. (a) Meningeal inflammation (hematoxylin and eosin stain, original magnification $\times 25$ ). (b) Rare intracellular cocci in the meninges (immunohistochemical assays with the N. meningitidis polyclonal antibody detected with naphthol fast red; original magnification $\times 157.5$ ). 
silver stains and immunohistochemistry, but in a minority of cases the meninges showed inflammation without showing the bacteria. Not surprisingly, more than half of the cases in this series were due to $S$. pneumoniae and $N$. meningitidis. In the US, vaccination for $H$. influenzae type b since the 1980 s has significantly decreased the frequency of acute bacterial meningitis due to this microorganism. ${ }^{3}$ In 2008 in Taiwan, the carriage of $H$. influenzae type b in children less than 5 years old was only $0.6 \%$ due to vaccination. ${ }^{24}$ Vaccines against $S$. pneumoniae and $N$. meningitidis are now recommended for children in the US, ${ }^{3}$ while Mexico and Taiwan have tested the immunogenicity and safety of providing at the same time the 10 -valent pneumococcal $H$. influenzae protein $\mathrm{D}$ conjugate vaccine, the tetravalent meningococcal vaccine, and tetanus toxoid conjugate vaccine in toddlers. ${ }^{25}$ It is logical to postulate that vaccination against these organisms may further decrease the mortality due to acute bacterial meningitis and pneumococcal pneumonia. Even though the current $S$. pneumoniae and $N$. meningitidis vaccines do not cover for all the different strains of these bacteria, the amount of coverage may work similar to what has happened with $H$. influenzae, where the vaccine only covers for type b still there has been a dramatic decrease of $H$. influenzae meningitis.

We had six patients with Streptococcus spp. Diverse streptococci other than $S$. pneumoniae have been implicated in patients with meningitis, including $S$. agalactiae (group B streptococci), S. pyogenes (group A streptococci), and S. suis. ${ }^{3}$ S. agalactiae is usually observed in neonates and there were only two neonates in our series: one had $S$. pneumoniae with very prominent neuritis that extended into the eye, while the other neonate had gram-negative coccobacilli that we could not further identify. $S$. pyogenes meningitis can be a complication of acute disease with primary focus in the lung, skin, or other organs and can occur at any age. Meningitis due to $S$. suis has been found in patients that have contact with pigs. In our series, one of the patients could have had S. suis; however, the PCR could not define whether this was $S$. suis or $S$. bovis. This was a 12-year-old female who had symptoms for 3 days and was not hospitalized. Information regarding contact with pigs was not available. Thus, in these six patients, PCR and sequencing did not help to definitively elucidate the species of streptococci present owing to the conserved nature of 16SrRNA gene. Species-specific PCR assays, targeting the genes that are more variable, were also attempted in these cases, but they also were not able to amplify specific species. A study by $\mathrm{Xu}$ et $a l,{ }^{26}$ could not determine the species of streptococci in $8 \%$ of 413 specimens using 16S rDNA PCR in fresh CSF specimens. In our series the percentage of cases with Streptococcus spp. was higher (18\%), but it should be noted that we used formalin-fixed tissues, which are known to yield lower quality fragmented nucleic acids compared with fresh CSF specimens.

Meningitis due to staphylococci is usually associated with previous neurosurgical procedures or neurological shunts. ${ }^{3,6}$ In our series, the patient with $S$. aureus meningitis was a 53-year-old diabetic patient that had periprostatic and renal abscesses but no history of surgical interventions to the central nervous system. In this patient, it can be postulated that he had bacteremia or septicemia due to $S$. aureus, which seeded the meninges. The patient with Staphylococcus spp. was a 61-year-old from Taiwan who had undergone diagnostic brain biopsies for discrete lesions in the temporal and parietal lobes. Immunohistochemistry and PCR were able to define that the bacteria were not $S$. aureus in this case. Although less frequently, other staphylococci have been associated with meningitis after surgical central nervous system interventions. ${ }^{27}$

In our series, there was one case with Fusobacterium, a commensal of the oral cavity, which has occasionally been reported to cause meningitis particularly after manipulation of teeth (including cleaning) has occurred. ${ }^{28-30}$ The patient with Fusobacterium was a college student who presented with 6 days of fever, chills, neck pain, changes in voice, and difficulty in swallowing, all symptoms that suggest Lemierre's syndrome, which is usually caused by Fusobacterium although vessel thrombosis was not documented in this case. In our series, the last case where an etiologic agent was identified corresponded to a 68-year-old diabetic patient with urosepsis, in which enterococci were cultured post-mortem from the blood and CSF by the submitting facility. Enterococci have been implicated as the cause of spontaneous and postoperative meningitis in adults and children in almost similar proportions. ${ }^{31}$

In our series, no agent could be found in 15\% cases while $\mathrm{Xu}$ et al, ${ }^{26}$ did not find an etiologic agent in $32 \%$ of 413 fresh CSF specimens tested with $16 \mathrm{~S}$ rDNA PCR. Gram stains in our study gave some insights as to possible agents. In three cases, grampositive cocci were observed in the meninges, thus any streptococci, staphylococci, or enterococci could have been the cause. One of the cases corresponded to a 57-year-old that had a renal transplant and was in a motor vehicle accident. $\mathrm{He}$ suffered multiple fractures and seizures. The second case was 53-year-old male with a history of chronic alcohol abuse and cerebellar ataxia, who was found dead at home. The autopsy showed blunt force trauma causing extensive retroperitoneal and mesenteric hemorrhage and microscopic evidence of meningitis. The history received for the third cases was minimal and included being a homeless person in Taiwan. For the two cases with gramnegative coccobacilli any of the enterobacteriaceae are potential etiologic agents. One patient was an 18-day-old neonate with failure to thrive. Meningitis 
due to gram-negative bacteria is more frequent in the neonatal period since babies are infected when they pass through the vaginal canal, which is colonized with enteric flora. The second case corresponded to a 13-year-old female who presented with fevers, headache, and change in mental status. This patient's demise occurred at the beginning of the influenza season and was originally attributed to influenza. Lastly, no organisms could be identified with any of the staining or molecular techniques in one case. We know this patient was a child but no history was provided.

For this study we used, for the most part, a tier approach where the results of the Gram and bacterial silver impregnation stains defined subsequent immunohistochemistry testing. Then the results of the immunohistochemistry testing defined the PCR testing to be performed (Figure 3). This approach can be useful in resource allocation but it may not be as easily followed in emergency situations, such as the need to start exposure prophylaxis for $N$. meningitidis contacts even when the index patient has expired. Although, it should be noted that both Gram and bacterial silver stains can be insensitive if the patient has received antibiotics. Immunohistochemistry has proven to be very valuable for organism-specific diagnosis in a variety of situations where there is suspicion of infectious diseases. The technique utilizes samples that are routinely obtained for histopathologic diagnosis, the tissues are non-infectious because of the formalin fixation, and the method has been largely automated. However, barriers to more widespread use of immunohistochemistry exist due to the lack of commercial antibodies and the need to validate these assays in each institution as there are no FDA approved kits. Similar to what happens with immunohistochemistry, PCR using formalinfixed, paraffin-embedded material was very useful in confirming the presence of the different organisms in this study; however, barriers similar to those present for immunohistochemistry are frequent for PCR. ${ }^{32}$

The pathology that was observed in our cases is similar to what has been described previously for $S$. pneumoniae except that thrombosis and vessel wall inflammation was not particularly evident. ${ }^{11,12}$ Inadequate sampling of the central nervous system is a possible explanation for missing areas of thrombosis and arteritis. Studies using magnetic resonance imaging (MRI) have demonstrated that the vessels most frequently affected are at the base of the brain. ${ }^{33}$ The cortical neuronal damage has been attributed not only to the inflammatory infiltrate but also to direct neuronal damage by bacterial cell components. $^{34}$ Parenchymal damage has been reported by using MRI in 7 of the 68 patients with acute bacterial meningitis. ${ }^{33}$

This study has several limitations and potential biases. Cases referred to the CDC for testing do not necessarily reflect all fatal bacterial meningitis cases seen in a particular population but reflect severe

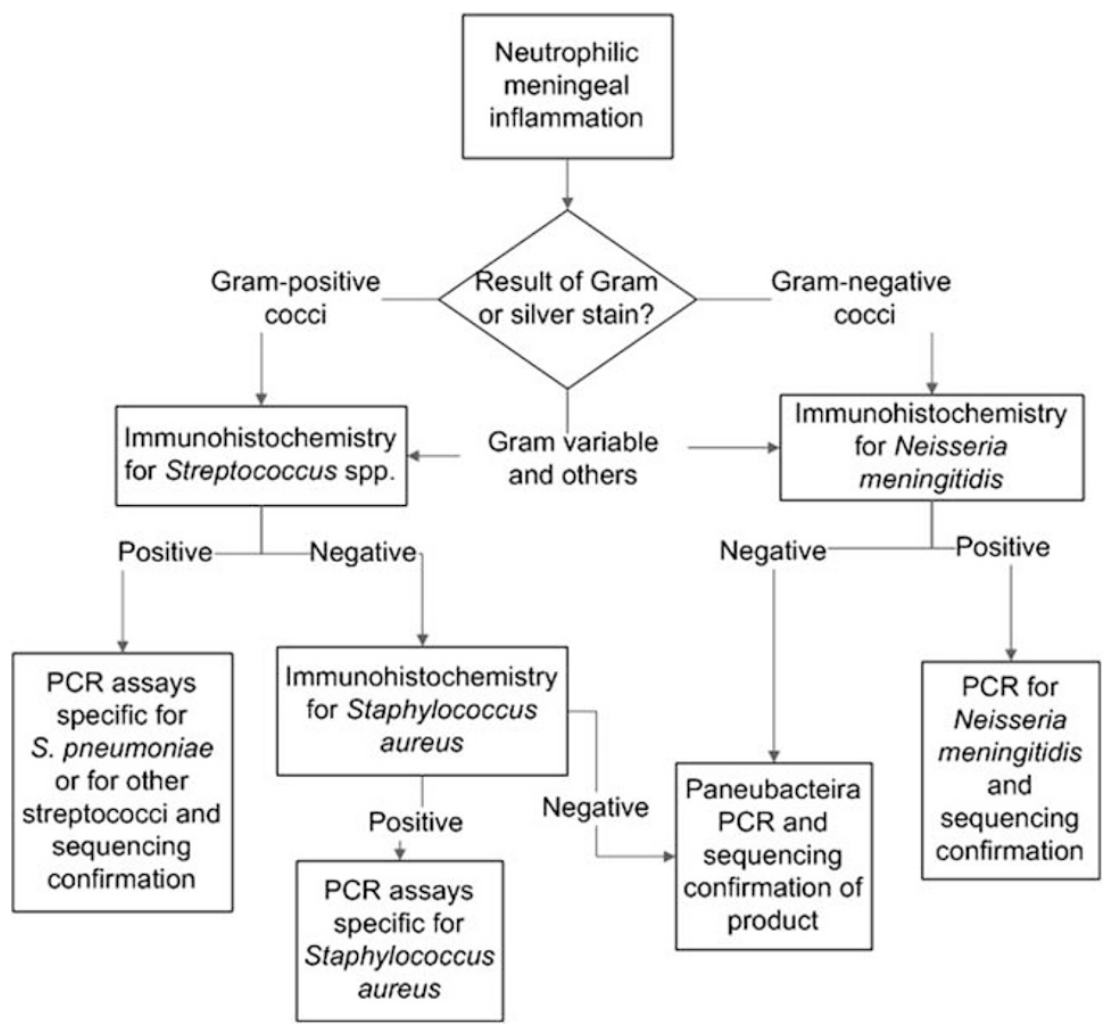

Figure 3 Work flow of specimens to define immunohistochemical and PCR testing to be performed. 
cases where an autopsy was obtained, and there is physician or public health interest in identifying an organism that may or may not have been suspected. Another limitation is the fact that the pathologists referring the case only sent selected tissue blocks, which does not allow for generalization of the histopathological findings and related varied amounts of clinical information. A third limitation is the fact that all the tissues were formalinfixed causing cross-linking of DNA and proteins, and degradation of nucleic acids, which did not allow for the amplification of large segments of DNA for organism-specific diagnosis in a percentage of cases.

In summary, immunohistochemistry and PCR performed on formalin-fixed, paraffin-embedded samples were instrumental in defining organismspecific diagnosis in fatal cases with acute meningeal inflammation and clinical suspicion of bacterial infection. S. pneumoniae continues to be the most frequent cause of fatal acute bacterial meningitis followed by $N$. meningitidis, thus vaccination for streptococcus and meningococcus at a younger age may prevent fatal cases.

\section{Acknowledgements}

We acknowledge all the state and local health departments and laboratories and all pathologists and medical examiners who submitted specimens to the Infectious Diseases Pathology Branch. We also thank Anne Whitney for her help in Neisseria PCR testing of some cases and Jackie Sampson for PCR testing for $S$. pneumoniae in a post-mortem CSF sample. We also greatly acknowledge our colleagues in the Infectious Disease Pathology Branch, especially Wun-Ju Shieh and Christopher D Paddock for their invaluable pathology expertise in evaluating cases and Jeltley Montague and Jeanine Bartlett for their technical assistance with special stains.

\section{Disclosure/conflict of interest}

The authors declare no conflict of interest.

\section{References}

1 Honda H, Warren D. Central nervous system infections: Meningitis and brain abscess. Infect Dis Clin N Am 2009;23:609-623.

2 Attia J, Hatala R, Cook D, et al. Does this adult patient have acute meningitis? JAMA 1999;282:175-181.

3 Brouwer M, Tunkel A, van-de-Beek D. Epidemiology, diagnosis, and antimicrobial treatment of acute bacterial meningitis. Clin Microbiol Rev 2010;23:467-492.

4 Cho H, Lee H, Kang J, et al. The causative organisms of bacterial meningitis in Korean children in 1996-2005. J Korean Med Sci 2010;25:895-899.
5 Moon S, Chung D, Kim S, et al. Changing etiology of community-acquired bacterial meningitis in adults: a nationwide multicenter study in Korea. Eur J Clin Microbiol Infect Dis 2010;29:793-800.

6 Huang W, Lee C, Liu J. Clinical characteristics and risk factors for mortality in patients with meningitis caused by Staphylococcus aureus and vancomycin minimal inhibitory concentrations against these isolates. J Microbiol Immunol Infect 2010;43:470-477.

7 Pérez A, Dickinson F, Rodríguez M. Community acquired bacterial meningitis in Cuba: a follow up of a decade. BMC Infect Dis 2010;10:130-139.

8 Gurley E, Hossain M, Montgomery S, et al. Etiologies of bacterial meningitis in Bangladesh: results from a hospital-based study. Am J Trop Med Hyg 2009; 81:475-483.

9 Lucey J, Gavin P, Cafferkey M, et al. Pneumococcal meningitis: clinical outcomes in a pre-vaccine era at a Dublin paediatric hospital, 1999-2007. Ir J Med Sci 2010;180:47-50.

10 Thigpen M, Whitney C, Messonnier N, et al. Bacterial meningitis in the United States, 1998-2007. N Engl J Med 2011;364:2016-2025.

11 Andersen C. Streptococcus penumoniae meningitis.clinical and experimental studies. Dan Med Bull 2007;54:189-209.

12 Leib S, Täuber M. Pathogenesis of bacterial meningitis. Infect Dis Clin North Am 1999;13:527-548.

13 Guarner J, Packard M, Nolte K, et al. Utility of immunohistochemical diagnosis of Streptococcus pneumoniae in formalin-fixed, paraffin-embedded specimens compared to culture and Gram's stains techniques. Am J Clin Pathol 2006;127:612-618.

14 Guarner J, Greer P, Whitney A, et al. Pathogenesis and diagnosis of human meningococcal disease using immunohistochemical and PCR assays. Am J Clin Pathol 2004;122:754-764.

15 Guarner J, Bartlett J, Reagan S, et al. Immunohistochemical evidence of Clostridium sp., Staphylococcus aureus, and group A streptococcus in severe soft tissue infections related to injection drug use. Hum Pathol 2006;37:1482-1488.

16 Guarner J, Sumner J, Paddock C, et al. Diagnosis of invasive group A streptococcal infections by using immunohistochemical and molecular assays. Am J Clin Pathol 2006;126:148-155.

17 Guarner J, Paddock C, Bartlett J, et al. Adrenal gland hemorrhage in patients with fatal bacterial infections. Mod Pathol 2008;21:1113-1120.

18 Shieh W, Blau D, Denison A, et al. 2009 pandemic influenza A (H1N1): pathology and pathogenesis of 100 fatal cases in the United States. Am J Pathol 2012;177:166-175.

19 Guarner J, Bhatnagar J, Shieh W, et al. Histopathologic, immunohistochemical, and polymerase chain reaction assays in the study of cases with fatal sporadic myocarditis. Hum Pathol 2007;38: 1412-1419.

20 Murdoch D, Anderson T, Beynon K, et al. Evaluation of a PCR assay for detection of Streptococcus pneumoniae in respiratory and nonrespiratory samples from adults with community-acquired pneumonia. J Clin Microbiol 2003;41:63-66.

21 Joosse S, van-Beers E, Tielen I, et al. Prediction of BRCA1-association in hereditary non-BRCA1/2 breast carcinomas with array-CGH. Breast Cancer Res Treat 2009;116:479-489. 
22 Lansac N, Picard F, Ménard C, et al. Novel genusspecific PCR-based assays for rapid identification of Neisseria species and Neisseria meningitidis. Eur J Clin Microbiol Infect Dis 2000;19:443-451.

23 Imrit K, Goldfischer M, Wang J, et al. Identification of bacteria in formalin-fixed, paraffin-embedded heart valve tissue via $16 \mathrm{~S}$ rRNA gene nucleotide sequencing. J Clin Microbiol 2006;44:2609-2611.

24 Wang S, Lo W, Chou C, et al. Low rate of nasopharyngeal carriage and high rate of ampicillin resistance for Haemophilus influenzae among healthy children younger than 5 years old in northern Taiwan. J Microbiol Immunol Infect 2008;41:32-40.

25 Ruiz-Palacios GM, Huang LM, Lin T, et al. Immunogenicity and safety of a booster dose of the 10-valent pneumococcal Haemophilus influenzae protein D conjugate vaccine co-administered with the tetravalent meningococcal serogroups A, C, W-135, and Y tetanus toxoid conjugate vaccine in toddlers: a randomized trial. Pediatr Infect Dis J 2013;32:62-71.

$26 \mathrm{Xu}$ J, Moore J, Millar B, et al. Employment of broad range $16 \mathrm{~S}$ RDNA PCR and sequencing in the detection of aetiological agents of meningitis. New Microbiol 2005;28:141-149.

27 Rothman R, Ramachandran P, Yang S, et al. Use of quantitative broad-based polymerase chain reaction for detection and identification of common bacterial pathogens in cerebrospinal fluid. Acad Emerg Med 2010;17:741-747.

28 Kutlu S, Sacar S, Cevahir N, et al. Communityacquired Streptococcus mitis meningitis: a case report. Int J Infect Dis 2008;12:e107-e109.

29 Moller K, Frederiksen E, Wandall J, et al. Meningitis caused by streptococci other than Streptococcus pneumoniae: a retrospective clinical study. Scand J Infect Dis 1999;31:375-381.

30 Le-Monnier A, Jamet A, Carbonnelle E, et al. Fusobacterium necrophorum middle ear infections in children and related complications: report of 25 cases and literature review. Pediatr Infect Dis J. 2008;27: 613-617.

31 Pintado V, Cabellos C, Moreno S, et al. Enterococcal meningitis: a clinical study of 39 cases and review of the literature. Medicine (Baltimore) 2003;82:346-364.

32 Burd E. Validation of laboratory-developed molecular assays for infectious diseases. Clin Microbiol Rev 2010;23:550-576.

33 Katchanov J, Siebert E, Endres M, et al. Focal parenchymal lesions in community-acquired bacterial meningitis in adults: a clinico-radiological study. Neuroradiol 2009;51:723-729.

34 Hoffmann O, Braun J, Becker D, et al. TLR2 mediates neuroinflammation and neuronal damage. J Immunol. 2007;178:6476-6481. 